\title{
Association of mannose binding lectin (MBL) gene polymorphism and serum MBL concentration with characteristics and progression of systemic lupus erythematosus
}

\author{
R Takahashi, A Tsutsumi, K Ohtani, Y Muraki, D Goto, I Matsumoto, N Wakamiya, T Sumida
}

Ann Rheum Dis 2005;64:311-314. doi: 10.1136/ard.2003.020172

Objective: To determine whether occurrence, characteristics, and progression of systemic lupus erythematosus (SLE) are associated with polymorphism of the mannose binding lectin (MBL) gene and with serum MBL concentration.

Methods: Codon $54 \mathrm{MBL}$ gene polymorphism of 147 patients with SLE and 160 healthy controls was determined by polymerase chain reaction-restriction fragment length polymorphism. Serum concentration of MBL was measured by enzyme immunoassay. Fluctuations of serum MBL were analysed with respect to disease characteristics and activity. Results: Frequency of homozygosity for codon 54 minority allele was $6 \%$ (9/147) in patients with SLE, and significantly higher than in controls ( $p=0.0294$, Fisher's exact test). MBL polymorphism in patients with SLE was not significantly associated with disease characteristics or immunological phenotypes. Patients homozygous for the B allele tended to have a higher risk of infection during treatment. Levels of C3 and $\mathrm{CH}_{50}$ were slightly, but significantly, associated with serum MBL concentration in patients with SLE homozygous for the majority allele. During the course of SLE, serum MBL concentration increased in $6 / 14$ patients, and decreased in 7 after initiation of immunosuppressive treatment.

Conclusions: MBL gene polymorphism influences susceptibility to SLE, but has no direct effect on disease characteristics. Serum MBL levels fluctuate during the course of SLE in individual patients. MBL genotyping may be useful in assessing the risk of infection during treatment of SLE.

M annose binding lectin (MBL) is a molecule that shares many features with Clq. MBL comprises a trimer of three identical polypeptides, and several trimers further combine to form a bouquet-like structure. ${ }^{1} \mathrm{MBL}$ mediates lectin dependent activation of the complement pathway, ${ }^{1}$ and has an important role in host defence against micro-organisms. People lacking this protein could develop severe episodes of bacterial infections from early life. ${ }^{2}$ Several polymorphisms have been reported for the MBL gene, and a large interindividual difference in serum MBL concentration is caused by the possession of variant alleles. Codon 52, 54, and 57 polymorphisms are all on exon 1 of the MBL gene, and the presence of any of the minority alleles significantly reduces serum MBL concentration. Furthermore, homozygosity for minority alleles results in almost complete deficiency of serum MBL. ${ }^{3}$ This has been attributed to increased degradation of the mutated protein. ${ }^{4}$

Recently, several studies have suggested that possession of MBL minority alleles may be associated with occurrence of systemic lupus erythematosus (SLE). ${ }^{5}$ It is known that Clq deficiency is associated with severe symptoms of SLE. ${ }^{7}$ Two possible explanations for associations between MBL or Clq deficiency and occurrence of SLE can be proposed: (a) MBL and Clq can bind to and initiate uptake of apoptotic cells into macrophages, ${ }^{89}$ and abnormal clearance of apoptotic cells caused by MBL or Clq deficiency may result in overexpression of autoantigens; $(b)$ viral infection is believed to be one of the causes of SLE, ${ }^{10}$ and MBL or Clq deficiency may lead to more frequent infections.

This study was conducted on the premise that occurrence, characteristics, and progression of SLE are associated with polymorphism of the MBL gene and with serum MBL concentration. To our knowledge, this is the first study that has measured serum MBL concentration before and after immunosuppressive treatment in patients with newly diagnosed SLE.

\section{PATIENTS AND METHODS}

Samples from 147 Japanese patients with SLE followed up at our hospital, were used for the study. All patients fulfilled the 1997 American College of Rheumatology Classification Criteria for SLE. Samples from 160 Japanese healthy volunteers served as controls.

Genomic DNA was purified from peripheral blood leucocytes using the DNAQuick DNA purification kit (Dainippon Pharmaceuticals, Osaka, Japan), and stored at $-30^{\circ} \mathrm{C}$. Typing of the MBL gene allele was performed by polymerase chain reaction-restriction fragment length polymorphism, according to the method of Madsen et al. ${ }^{3}$ The wild-type allele was designated allele $\mathrm{A}$, and codon 54 substitution (glycine to aspartic acid) was designated allele B. Previous studies have shown that codon 52 and 57 polymorphisms are not present or extremely rare in the Japanese population. ${ }^{11}$ Serum concentration of MBL was measured by a specific enzyme immunoassay using two rabbit polyclonal anti-MBL antibodies as described previously. ${ }^{12}$

Table 1 Codon 54 genotypes of the MBL gene in patients with SLE and healthy controls

\begin{tabular}{llll}
\hline & SLE & Healthy controls & $p$ Value \\
\hline$A A+A B$ & 138 & 158 & \\
& (AA; 84, AB; 54) & (AA; 101, AB; 57) & \\
& 9 & 2 & 0.0294 \\
$B B$ & 9 & 160 & \\
Total & 147 & & \\
\hline
\end{tabular}

Allele $\mathrm{A}$, codon 54 wild type majority allele; allele B, codon 54 variant minority allele.

$\mathrm{p}$ Value by Fisher's exact test. 


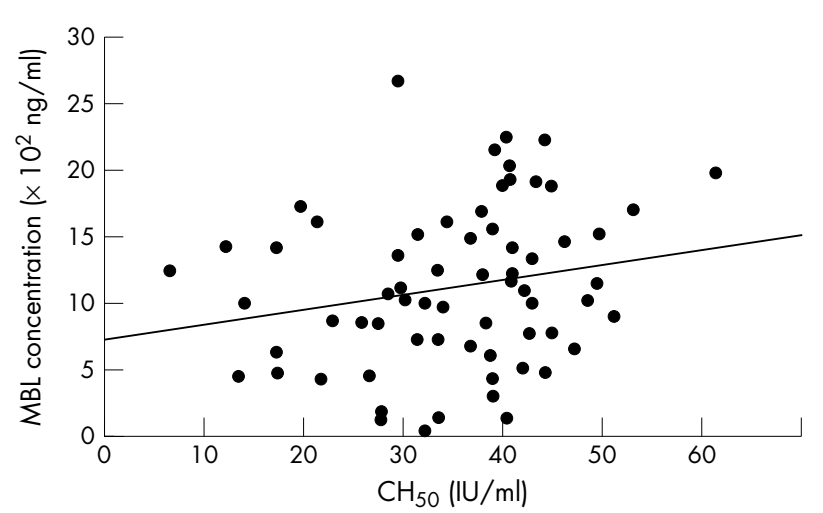

Figure 1 Relationship between serum $\mathrm{MBL}$ concentration and $\mathrm{CH}_{50}$ in genotype AA patients with SLE. $r_{s}=0.253, p=0.0412$ by Spearman's rank correlation test.

Fisher's exact test was used to compare the frequencies of genotypes $\mathrm{AA} / \mathrm{AB}$ and $\mathrm{BB}$, between disease and control groups, and to compare clinical characteristics between patients with genotypes $\mathrm{AA} / \mathrm{AB}$ and those with $\mathrm{BB}$. MannWhitney's U test was used to compare ages at diagnosis of SLE between patients with genotypes AA/AB and those with $\mathrm{BB}$, and to compare serum MBL concentration between patients and controls of the same genotype. Spearman's rank correlation test was used to compare serum MBL concentration and the levels of anti-DNA antibody, C3, C4, and $\mathrm{CH}_{50}$. Values of $\mathrm{p} \leqslant 0.05$ were considered significant.

\section{RESULTS}

MBL gene genotypes were studied in patients with SLE and healthy controls (table 1). Among 147 patients with SLE, 9 were homozygous for allele $\mathrm{B}$, which was significantly increased compared with controls $(p=0.0294)$.

We analysed the difference in disease characteristics among patients with SLE categorised by MBL genotypes. Ages (mean (SD)) at diagnosis of SLE tended to be younger in patients with allele B (AA: 32.5 (14.8); AB: 30.7 (15.2); BB: $23.4(13.3))$, but no significant differences were seen $(p=0.0681)$. Clinical characteristics, serological, and immunological measures did not significantly differ between genotype BB patients and other patients with SLE. This is
A
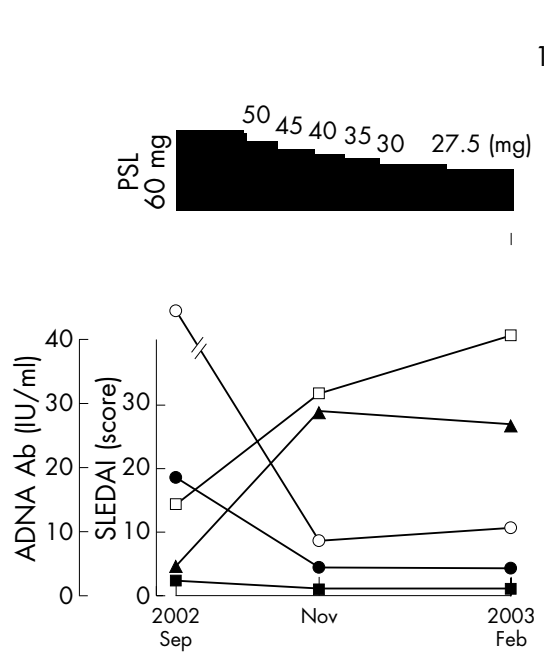

C
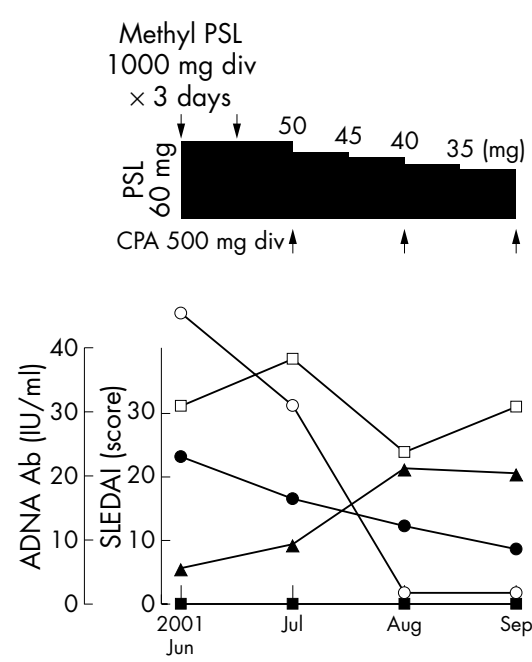

B
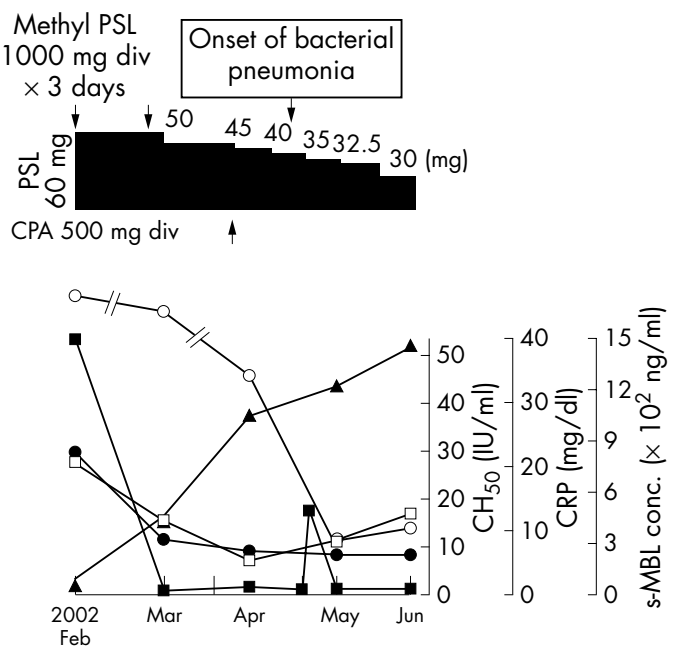

D

Methyl PSL

$1000 \mathrm{mg}$ div

$\times 3$ days
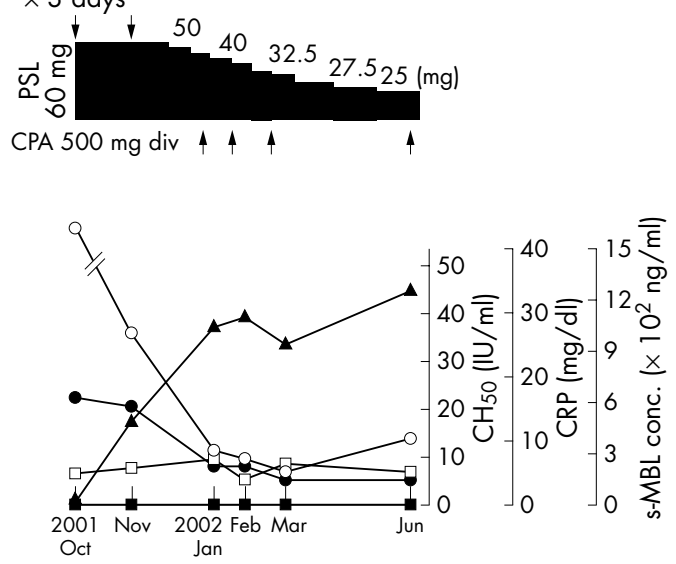

Figure 2 Fluctuation of serum MBL concentration and clinical variables during immunosuppressive treatment in patients with newly diagnosed SLE. Open squares, serum MBL concentrations (s-MBL); closed squares, $C$ reactive protein (CRP); open circles, anti-DNA antibody (ADNA Ab); closed circles, SLE Disease Activity Index (SLEDAI); closed triangles: $\mathrm{CH}_{50}$. PSL, prednisolone; CPA, cyclophosphamide. 
most probably because of the small size of the BB cohort. However, incidence of infections requiring admission to hospital was significantly higher in patients with genotype $\mathrm{BB}$ than in other patients (genotype $\mathrm{AA}+\mathrm{AB} ; 35 / 132$ patients, $\mathrm{BB} ; 5 / 8$ patients, $\mathrm{p}=0.0287$ ).

Serum MBL concentration reflected the MBL genotype of the individual subject, in accordance with previous reports ${ }^{3}$ (data not shown). Among subjects with the same genotype, patients with SLE tended to have a higher MBL concentration than controls, but without statistical significance. The level of $\mathrm{CH}_{50}$ was weakly but significantly associated with serum MBL concentration in patients with SLE with genotype AA $(\mathrm{p}=0.0412)$ (fig 1$)$. In genotype AA patients, C3 was also associated with serum MBL concentration, although $\mathrm{C} 4$ was not $(C 3 ; p=0.0494, C 4 ; p=0.4265)$. No significant relationship between anti-DNA antibody titre and serum MBL was found. In patients with other genotypes, no significant association was seen between serum MBL concentration and levels of anti-DNA antibody or complement components (data not shown).

We studied fluctuation of serum MBL concentration during immunosuppressive treatment in patients with newly diagnosed SLE (fig 2). In patient 1 with genotype AA (fig 2A), serum MBL increased in parallel with $\mathrm{CH}_{50}$ after initiation of methylprednisolone treatment, while the SLE Disease Activity Index (SLEDAI) and anti-DNA antibody decreased. In patient 2 with genotype AA (fig 2B), serum MBL concentration decreased after initiation of methylprednisolone pulse therapy, while $\mathrm{CH}_{50}$ increased. After CRP decreased to normal levels, MBL gradually increased in parallel with $\mathrm{CH}_{50}$. In patient 3 with genotype AA (fig 2C), serum MBL did not show a clear trend, although disease activity steadily decreased. In patient 4 with genotype $A B$ (fig 2D), serum MBL was low throughout, reflecting the MBL genotype. When the serum MBL concentration before and after immunosuppressive treatment was compared in 14 newly diagnosed patients, it increased in 6/14 patients (genotype AA: 2, AB: 4), and decreased in 7 patients (genotype AA: 5, AB: 2). There was no significant association between increase or decrease of serum MBL concentration and genotypes and clinical phenotypes in patients with SLE (data not shown).

\section{DISCUSSION}

Several studies have indicated that MBL gene polymorphism influences susceptibility to SLE. ${ }^{56}$ When the components of the classical pathway of complement (Clq, Clr, Cls, C4, or C2) are deficient, it has been suggested that abnormal clearance of not only immune complexes ${ }^{13}$ but also apoptotic cells $^{8}$ contributes to the occurrence of SLE. It has been indicated that inappropriate levels of apoptotic nuclei may be a major source of autoantigens in SLE. ${ }^{14}$ Recently, it was reported that MBL can bind to apoptotic cells and initiate their uptake by macrophages, ${ }^{9}$ and thus, abnormal clearance of apoptotic cells due to MBL deficiency may provide a source of autoantigens in SLE. However, deficiency of MBL is not an extremely high risk factor, in contrast with deficiencies of other complement molecules such as $\mathrm{Clq}^{7}$ The precise consequences of MBL deficiency for the onset and progression of SLE remain unclear. The lag time between occurrence of the first symptom attributable to SLE and diagnosis of definite SLE was reported to be significantly shorter for variant allele carriers than in those with genotype AA. ${ }^{6}$ Therefore, the MBL gene may be a disease modifier locus rather than a true SLE susceptibility locus. Although no significant correlation between disease characteristics and MBL genotypes was seen, genotype $\mathrm{BB}$ was significantly associated with occurrence of infection in our patients, in accord with a previous report. ${ }^{6}$ MBL genotyping may help in assessment of the risk of opportunistic infections in patients with SLE.

The balance of MBL production and consumption determines serum MBL levels. As the presence of MBL deposits in tissues of autoimmune patients has been demonstrated, ${ }^{15} 16$ we expected that MBL would be consumed during active disease, and that serum MBL concentration might reflect disease activity and pathological features of SLE in individual patients. To test this hypothesis, we measured serum MBL concentration during immunosuppressive treatment in patients with newly diagnosed disease. As shown in fig 2, serum MBL concentration did fluctuate during the course of immunosuppressive treatment in patients with SLE, especially in genotype AA patients. In patients 1 and 2, the increasing phase of serum MBL concentration may reflect the decreased consumption of MBL while SLE activity gradually decreased, and the decreasing phase may reflect reduced production of MBL because MBL is an acute phase inflammatory protein. ${ }^{17}$ Thus, MBL levels appear to reflect disease activity in some patients. The weak but significant association between serum MBL concentration and serum C3 or $\mathrm{CH}_{50}$ levels supports this view.

In conclusion, frequency of homozygosity for a minority allele of the MBL gene was increased in patients with SLE compared with controls, confirming previous studies. MBL gene polymorphism may have no direct effect on disease characteristics, but patients homozygous for the minority allele had significantly more frequent episodes of infections. Serum MBL levels did fluctuate during the course of SLE in individual patients, although the mechanism of their fluctuation and their consequences in SLE are unclear. The value of serum MBL monitoring in clinical practice should be determined in future studies.

\section{Authors' affiliations}

R Takahashi, A Tsutsumi, Y Muraki, D Goto, I Matsumoto, T Sumida, Division of Rheumatology, Department of Internal Medicine, Institute of Clinical Medicine, University of Tsukuba, Japan

K Ohtani, N Wakamiya, Department of Microbiology, Asahikawa Medical College, Japan

Correspondence to: Dr A Tsutsumi, 1-1-1 Tennodai Tsukuba-city, Ibaraki 305-8575, Japan; atsutsum@md.tsukuba.ac.jp

\section{Accepted 9 May 2004}

\section{REFERENCES}

1 Holmskov U, Malhotra R, Sim RB, Jensenius JC. Collectins: collagenous $\mathrm{C}$-type lectins of the innate immune defense system. Immunol Today 1994; 15:67-74.

2 Summerfield JA, Ryder S, Sumiya M, Thursz M, Gorchein A, Monteil MA, et al. Mannose binding protein gene mutations associated with unusual and severe infections in adults. Lancet 1995:345:886-9.

3 Madsen HO, Garred P, Kurtzhals JA, Lamm LU, Ryder LP, Thiel S, et al. A new frequent allele is the missing link in the structural polymorphism of the human mannan-binding protein. Immunogenetics 1994;40:37-44.

4 Sumiya M, Super M, Tabona P, Levinsky RJ, Arai T, Turner MW, et al. Molecular basis of opsonic defect in immunodeficient children. Lancet 1991;29:1569-70.

5 Davies EJ, Snowden N, Hillarby MC, Carthy D, Grennan DM, Thomson W, et al. Mannose-binding protein gene polymorphism in systemic lupus erythematosus. Arthritis Rheum 1995;38:110-14.

6 Garred P, Voss A, Madsen HO, Junker P. Association of mannose-binding lectin gene variation with disease severity and infections in a population-based cohort of systemic lupus erythematosus patients. Genes Immun 2001;2:442-50.

7 Walport MJ, Davies KA, Botto M. Clq and systemic lupus erythematosus. Immunobiology 1998;199:265-85.

8 Korb LC, Ahearn JM. Clq binds directly and specifically to surface blebs of apoptotic human keratinocytes: complement deficiency and systemic lupus erythematosus revisited. J Immunol 1997;158:4525-8.

9 Ogden CA, deCathelineau A, Hoffmann PR, Bratton D, Ghebrehiwet B, Fadok VA, et al. $\mathrm{Cl} q$ and mannose binding lectin engagement of cell surface calreticulin and CD91 initiates macropinocytosis and uptake of apoptotic cells. J Exp Med 2001;194:781-95. 
10 Okada M, Ogasawara H, Kaneko H, Hishikawa T, Sekigawa I, Hashimoto H, et al. Role of DNA methylation in transcription of human endogenous retrovirus in the pathogenesis of systemic lupus erythematosus. J Rheumatol 2002;29:1678-82

11 Sasaki K, Tsutsumi A, Wakamiya N, Ohtani K, Suzuki Y, Watanabe Y, et al. Mannose-binding lectin polymorphisms in patients with hepatitis $C$ virus infection. Scand J Gastroenterol 2000;35:960-5.

12 Ohtani K, Suzuki Y, Eda S, Kawai T, Kase T, Keshi H, et al. High-level and effective production of human mannan-binding lectin $(\mathrm{MBL})$ in Chinese hamster ovary (CHO) cells. J Immunol Methods 1999;222:135-44.

13 Atkinson JP. Complement deficiency: predisposing factor to autoimmune syndromes. Clin Exp Rheumatol 1989;7:S95-101.
14 Mevorach D, Zhou JL, Song X, Elkon KB. Systemic exposure to irradiated apoptotic cells induces autoantibody production. J Exp Med 1998; 188:387-92.

15 Hisano S, Matsushita M, Fujita T, Endo Y, Takebayashi S. Mesangial IgA2 deposits and lectin pathway-mediated complement activation in $\lg \mathrm{A}$ glomerulonephritis. Am J Kidney Dis 2001;38:1082-8.

16 Steinfeld S, Penaloza A, Ribai P, Decaestecker C, Danguy A, Gabius HJ, et al. $\mathrm{D}$-mannose and $\mathrm{N}$-acetylglucosamine moieties and their respective binding sites in salivary glands of Siögren's syndrome. J Rheumatol 1999;26:833-41.

17 Thiel S, Holmskov U, Hviid L, Laursen SB, Jensenius JC. The concentration of the $\mathrm{C}$-type lectin, mannan-binding protein, in human plasma increases during an acute phase response. Clin Exp Immunol 1992;90:31-5.

\section{Get published within days of acceptance with ARD}

We are delighted to announce that the Annals of the Rheumatic Diseases launched a "publish ahead of print" programme in February 2004. Selected papers are fast tracked and published online months before they appear in the print journal.

Papers of major significance to the international rheumatology community are published within days of acceptance. The first published article is the raw accepted manuscript; edited and typeset versions are also published as soon as they are available.

In addition to being available on ARD Online, the publish ahead of print articles are searchable through PubMed/ Medline-establishing primacy for your work. They are linked from the ARD Online home page.

$A R D$ 's publish ahead of print programme is unique among the major rheumatology journals - to take advantage of this service submit your papers to Annals of the Rheumatic Diseases using our online submission and review system Bench>Press (http://submit-ard. bmijournals.com). For further information contact ARD@bmigroup.com. 